\title{
Pneumocystis-jiroveci-Pneumonie (PcP) bei Patienten mit rheumatologischen Erkrankungen: Fallbeschreibung und Review
}

Bertisch, B ; Ruef, C

\begin{abstract}
Eine 74-jährige, polymorbide Patientin mit einer rheumatoiden Arthritis erlitt unter Therapie mit Methotrexat und Prednison eine Pneumocystis-jiroveci-Pneumonie (PcP). Eine Therapie mit Bactrim wurde eingeleitet. Trotz nicht mehr nachweisbaren Pneumozysten in einer Bronchiallavage verstarb die Patientin. Die genaue Todesursache blieb unklar. Wie dieses Beispiel zeigt, muss bei immunkompromittierten rheumatologischen Patienten an die Differenzialdiagnose einer PcP gedacht werden. Der typische Verlauf, Diagnose, Prophylaxe und Therapie der PcP bei dieser Patientengruppe werden diskutiert
\end{abstract}

DOI: https://doi.org/10.1007/s00393-005-0019-y

Posted at the Zurich Open Repository and Archive, University of Zurich

ZORA URL: https://doi.org/10.5167/uzh-155903

Journal Article

Published Version

Originally published at:

Bertisch, B; Ruef, C (2006). Pneumocystis-jiroveci-Pneumonie (PcP) bei Patienten mit rheumatologischen Erkrankungen: Fallbeschreibung und Review. Zeitschrift für Rheumatologie, 65(1):18-23.

DOI: https://doi.org/10.1007/s00393-005-0019-y 
Z Rheumatol 2006 $65: 18-23$ DOI 10.1007/s00393-005-0019-y

Online publiziert: 19. Januar 2006

(c) Springer Medizin Verlag 2006

\author{
B. Bertisch ${ }^{1} \cdot$ C. Ruef ${ }^{1,2}$ \\ ${ }^{1}$ Klinik für Infektionskrankheiten und Spitalhygiene, Departement für Innere Medizin, \\ Universitätsspital Zürich \\ ${ }^{2}$ Klinik für Infektionskrankheiten und Spitalhygiene, Departement für Innere Medizin, \\ Universitätsspital Zürich
}

\section{Pneumocystis-jiroveci- Pneumonie (PCP) bei Patienten mit rheumato- logischen Erkrankungen} Fallbeschreibung und Review

\section{Kasuistik}

Bei einer 74-jährigen Patientin war seit 2001 eine rheumatoide Arthritis mit positivem Rheumafaktor bekannt. Eine Basistherapie mit Methotrexat wurde ab Januar 2003 durchgeführt und lediglich kurzzeitig wegen zunehmender Dyspnoe bei möglicher Lungenfibrose unterbrochen. Zwischenzeitliche Monotherapien mit Prednison und Imurek waren nicht ausreichend wirksam. Zwei Monate vor der stationären Aufnahme wurde das Methotrexat bei Dyspnoe erneut abgesetzt, in der Folge wurde die Therapie mit Prednison $10 \mathrm{mg} /$ Tag durchgeführt.

Die aktuelle stationäre Einweisung erfolgte wegen exazerbierter Polyarthritis mit sekundärer, aktivierter Gonarthrose rechts. Es bestand eine Morgensteifigkeit von 1-2 Stunden mit Beteiligung der Schulter-, Hand- und Fingergrundgelenke sowie des rechten Knies. Eine B-Symptomatik zeigte sich nicht. Zudem waren 2 Tage zuvor eine Verschlechterung der Dyspnoe sowie thorakale Schmerzen links dorsobasal aufgetreten.

Bei der klinischen Untersuchung zeigte sich die Patientin in reduziertem Allgemeinzustand. Es fand sich ein Blutdruck von 140/100 mmHg, die Pulsfrequenz betrug 97/min. Der Auskultationsbefund der Lunge ergab beidseits basal diskontinuierliche Geräusche. Die Atemfrequenz betrug $25 / \mathrm{min}$ bei normaler Sauerstoffsättigung. An beiden Beinen fielen Ödeme auf. An den oben beschriebenen Gelenken fanden sich Zeichen einer Synovitis, am rechten Kniegelenk mit deutlichem Erguss und Überwärmung.

Laborchemisch zeigte sich eine deutlich erhöhte entzündliche Aktivität mit einem CRP von $246 \mathrm{mg} / \mathrm{l}$ und einer BSG von $78 \mathrm{~mm}$. Die Punktion des rechten Kniegelenks ergab einen entzündlichen Erguss ohne Nachweis von Bakterien.

Das rechte Knie sowie die rechte Schulter wurden mit Kortison infiltriert, die perorale Steroidgabe fortgeführt. Im Verlauf waren die Untersuchungsbefunde regredient. Das CRP sank 6 Tage nach Aufnahme auf $11 \mathrm{mg} / \mathrm{l}$.

Abklärungen bezüglich der Dyspnoe ergaben im konventionellen Röntgen basal betonte retikuläre Transparenzminderungen, in der Lungenfunktionsprüfung eine leichte Restriktion. Bei Zeichen der Herzinsuffizienz und Status nach einmaligem Vorhofflimmern wurde eine Herzinsuffizienztherapie eingeleitet. Die Dyspnoe sowie der initial erhöhte Pro-BNPWert verbesserten sich im Verlauf.

Zusätzlich zum Prednison wurde die Patientin 4 Tage nach Aufnahme erneut mit $15 \mathrm{mg}$ Methotrexat s. c. einmal wöchentlich behandelt. Wegen Anstiegs der Leberenzyme wurde dies nach zweimaliger Applikation wieder gestoppt.

18 Tage nach Spitaleintritt entwickelte die Patientin einen erneuten CRP-Anstieg bis über $200 \mathrm{mg} / \mathrm{l}$ sowie progrediente Dyspnoe mit nächtlichem Abfall der Sauerstoffsättigung unter $80 \%$. In der Computertomographie (CT) zeigten sich ausgedehnte milchglasartige Infiltrate der gesamten Lunge und vorwiegend basal betonte retikuläre Veränderungen. Die Bronchoskopie ergab eine chronische Tracheobronchitis mit mäßig viel leicht eitrigem Schleim. In der bronchoalveolären Lavageflüssigkeit fanden sich Pneumocystis jiroveci sowie Aspergillus fumigatus.

Eine Therapie mit Bactrim und hochdosiertem Prednison wurde eingeleitet. Bei respiratorischer Dekompensation wurde die Patientin auf die Intensivstation verlegt und intubiert. Im Verlauf kam es zu einer Verschlechterung mit akutem Lungenversagen, zunehmender Kreislaufinsuffizienz, beginnender Sepsis sowie Anstieg der Infektzeichen trotz adäquater Behandlung der PcP. Eine erneute Bronchoskopie ergab 6 Tage nach Start der Bactrim-Therapie weder Nachweis von Pneumocystis jiroveci noch Aspergillus, der Galactomannantest war nega- 
tiv. Bronchoskopisch fand sich Candida albicans. In einer Blutkultur waren Enterokokken nachweisbar.

Bei weiterer klinischer Verschlechterung wurde nach Rücksprache mit den Angehörigen von lebenserhaltenden Maßnahmen abgesehen, die Patientin verstarb. Die Durchführung einer Autopsie wurde von den Angehörigen abgelehnt.

\section{Diskussion}

Erstmals wurde 1942 eine Infektion mit Pneumocystis carinii beim Menschen beschrieben. Der Erreger wurde initial als Protozoon eingestuft, jüngere Daten hingegen zeigen, dass er näher mit den Pilzen verwandt ist [17]. Initial wurde der Erreger beim Menschen als Pneumocystis carinii bezeichnet. Aufgrund der genetischen und funktionalen Unterschiede zwischen Pneumozysten, die bei Mensch und Tier gefundenen werden, erfolgte eine Reklassifikation als Pneumocystis jiroveci. Die Wahrscheinlichkeit des Erwerbs einer Pneumocystis-jiroveci-Pneumonie $(\mathrm{PcP})$ ist neben den nachstehend beschriebenen Risiken auch abhängig von geographischen Faktoren und der untersuchten Institution [17]. Sowohl eine Reinfektion als auch die Reaktivierung einer latenten Infektion werden dabei als pathogenetische Mechanismen beschrieben [17].

\section{Wer erkrankt an einer PcP?}

Patienten mit einer zellulären Immunschwäche tragen ein erhöhtes Risiko, eine symptomatische Pneumocystisinfektion zu entwickeln [17]. Vor dem routinemäßigen Einsatz der PcP-Prophylaxe erkrankten die meisten HIV-infizierten Patienten mit fortgeschrittener Immundefizienz (<20o CD 4-Zellen/ $\mu \mathrm{l})$ an dieser Infektion. Auch nach Einführung der hoch aktiven antiretroviralen Therapien bleibt diese die häufigste Aidsdefinierende Erkrankung [6]. Ebenfalls ein erhöhtes Risiko haben Patienten mit malignen Erkrankungen, hämatopoetischen Stammzelltransplantationen, nach Transplantation solider Organe sowie Patienten, die immunsuppressive Therapien für Kollagenosen und Vaskulitiden erhalten [17].

Tabelle 1

Risikofaktoren für PcP bei rheumatologischen Erkrankungen

\begin{tabular}{ll} 
Medikament & Risiko \\
\hline Kortikosteroide & Ohne andere Immunsuppressiva: \\
& Risiko hoch bei Gabe von >30 mg [19] \\
& bzw. Risiko bei >20 mg, während 2-3 Wochen gegeben [4, 17] \\
& Mit anderen Immunsuppressiva: \\
& Risiko sogar bei <10 mg/Tag [19] \\
& Wahrscheinlich höheres Risiko bei Einsatz als tägliche Therapie \\
& bei Wegener-Granulomatose im Vergleich zu Pulstherapie bei \\
& rheumatoider Arthritis [21] \\
Zyklophosphamid & Ohne andere Immunsuppressiva: \\
& vereinzelte Berichte [5, 9, 11], dabei z. T. Niereninsuffizienz \\
berichtet [9] & Mit Kortikosteroiden: \\
Methotrexat & mehrfach berichtet [5, 9, 11, 14, 18, 22], dabei mindestens in \\
& einzelnen Fällen zusätzliche Gabe von NSAR \\
& Mit NSAR bzw. Aspirin: \\
& vereinzelte Berichte [9, 11, 22]
\end{tabular}

${ }^{a}$ Aufgrund der limitierten Fallzahlen sind Aussagen zur Risikoquantifizierung nur begrenzt möglich.

\section{PcP bei Patienten mit rheumatolo- gischen Erkrankungen}

In einem Kollektiv von Patienten mit PcP betrug der Anteil der Patienten mit Vaskulitis oder Autoimmunerkrankungen $22 \%$ [2]. Dabei waren Patienten mit Wegener-Granulomatose deutlich häufiger betroffen [2]; bei diesen wurde auch in anderen Studien ein erhöhtes Risiko mit bis zu $12 \%$ Erkrankungen berichtet $[5,15$, 17]. Die Inzidenz bei weiteren rheumatologischen Erkrankungen betrug in einer Studie mit 34 rheumatologischen $\mathrm{Pa}$ tienten $0,13 \%$ bei rheumatoider Arthritis. Dies entsprach bei einer weiteren Arbeitsgruppe 2 PcP-Fällen pro 10.000 Hospitalisationen pro Jahr [25], o,8\% bei systemischem Lupus erythematodes (SLE), 1,2\% bei Panarteriitis nodosa und $2 \%$ bei Polymyositis/Dermatomyositis [5]. Diese Angaben sind allerdings aufgrund der geringen Fallzahlen mit Vorsicht zu interpretieren. Zudem wurden Fälle von PcP beschrieben bei Riesenzellarteriitis, SharpSyndrom und Psoriasis-Arthritis [9, 26].

Dabei tritt die PcP fast ausschließlich unter Behandlung mit Immunsuppressiva auf, wie - Tabelle 1 zeigt. Das Auftreten einer $\mathrm{PcP}$ ohne immunsuppressive Therapie wurde selten berichtet (2 Fälle von SLE; [5]).

Neben einer PcP muss differenzialdiagnostisch auch an das Vorliegen weiterer infektiöser pulmonaler Erkrankungen gedacht werden. Bei einer nicht auf rheumatologische Patienten beschränkten Auswertung hatten sich in $35 \%$ der Fälle bakterielle Pneumonien oder Zytomegalievirus-Pneumonien gezeigt [2]. Unter immunsuppressiver Behandlung wurden in geringerer Anzahl auch weitere infektiologische Komplikationen wie Infektionen mit Mycobacterium avium, Histoplasma capsulatum in Endemiegebieten, Cryptococcus neoformans und Nocardia spp. beschrieben $[1,7,11]$. Eine Koinfektion mit PcP und Aspergillus wurde zweimal beschrieben: als nosokomiale Komplikation bei einem auf der Intensivstation behandelten Patienten [5] und als außerhalb des Krankenhauses erworbene Erkrankung [5, 14].

Bei Patienten mit rheumatologischen Erkrankungen hat sich seit 1980 eine $\mathrm{Zu}$ nahme der Fälle von PcP gezeigt. Dies könnte daraus resultieren, dass immunsuppressive Medikamente häufiger, in hö- 
Z Rheumatol 2006 $65: 18-23$

DOI 10.1007/s00393-005-0019-y

() Springer Medizin Verlag 2006

\section{B. Bertisch · C. Ruef}

Pneumocystis-jiroveci-Pneumonie (PcP) bei Patienten mit rheumatologischen Erkrankungen: Fallbeschreibung und Review

\section{Zusammenfassung}

Eine 74-jährige, polymorbide Patientin mit einer rheumatoiden Arthritis erlitt unter

Therapie mit Methotrexat und Prednison eine Pneumocystis-jiroveci-Pneumonie (PcP). Eine Therapie mit Bactrim wurde eingeleitet. Trotz nicht mehr nachweisbaren Pneumozysten in einer Bronchiallavage verstarb die Patientin. Die genaue Todesursache blieb unklar. Wie dieses Beispiel zeigt, muss bei immunkompromittierten

\section{Pneumocystis jiroveci pneumonia (PcP) in patients with rheumatic diseases: case report and review}

\begin{abstract}
A 74-year-old female patient with rheumatoid arthritis was diagnosed with Pneumocystis jiroveci pneumonia (PcP) following therapy with methotrexate and prednisone. Although bactrim treatment was initiated and PcP was not detected by a control bronchoalveolar lavage, the patient died. The precise cause of death remains unknown. As this case illustrates, PcP must be considered as a differential diagnosis in
\end{abstract}

rheumatologischen Patienten an die Differenzialdiagnose einer PcP gedacht werden. Der typische Verlauf, Diagnose, Prophylaxe und Therapie der PcP bei dieser Patientengruppe werden diskutiert.

\section{Schlüsselwörter}

Rheumatologische Erkrankungen · Pneumocystis-jiroveci-Pneumonie (PcP) $\cdot$ Immunsuppressive Therapie heren Dosierungen oder in Kombinationen verabreicht werden, die die Anfälligkeit der Patienten für PcP steigern könnten [25]. Die Hinzunahme neuer immunsuppressiv wirkender Medikamente könnte ebenfalls dazu beitragen. Beispielsweise wurde eine PcP bei zusätzlichem Einsatz des TNF- $\alpha$-Blockers Infliximab neben der Therapie mit Methotrexat und Kortikosteroiden beschrieben [24].

Zudem könnte die Zunahme der Fälle bedingt sein durch bessere Diagnosetechniken und höhere Vertrautheit mit dem Krankheitsbild [25].

Bei Patienten mit einer HIV-Infektion ist die Höhe der CD 4-Zellzahl ein Prädiktor für das PcP-Risiko. Bei HIV-negativen immunsupprimierten Patienten zeigen sich diesbezüglich unterschiedliche Resultate: Bei einer Studie hatten 91\% der PcP-Patienten CD 4- Zellen <3oo/ $\mu$, was einen Zusammenhang erahnen ließ [12], bei einer weiteren Studie fand sich dieses Verhältnis nicht [5].

\section{Verlauf der PcP bei HIV-negativen immunsupprimierten Patienten}

immunocompromised patients with rheumatic disease. The typical course, diagnosis, prophylaxis and treatment of PcP in this patient group are discussed.
Keywords
Rheumatic diseases - Pneumocystis jiro- veci pneumonia (PCP) - Immunosuppres- sive therapy

Bei einer Untersuchung an 34 Patienten mit Erkrankungen aus dem rheumatischen Formenkreis wurde die PcP in der Mehrheit (74\%) während der ersten 8 Monate der Erkrankung diagnostiziert [5, 17]. Die durchschnittliche Zeit vom Start der Symptome bis zur Diagnose betrug $6 \mathrm{Ta}$ ge [5]. In einer weiteren Studie betrug diese Zeit bei den untersuchten 37 HIV-negativen immunsupprimierten Patienten 5 Tage, hingegen bei den untersuchten 40 Aids-Patienten 28 Tage [8].

Typischerweise kommt es dabei zum Auftreten von progressiver Dyspnoe, Tachypnoe, Zyanose und nichtproduktivem Husten. Fieber, Schweißausbrüche oder grippeähnliche Symptome können hinzutreten [4].

Dabei scheint bei Nicht-Aids-Patienten der klinische Verlauf häufig schwerer zu sein. Sie zeigen eine ausgeprägtere Hypoxämie, und die Anzahl der Erreger ist im Allgemeinen geringer, was eine nichtinvasive Diagnose erschwert [4, 8]. Trotz signifikanter Hypoxämie und diffusem Parenchymbefall kann das Thorax-Röntgenbild komplett normal sein. Häufig aber werden diffuse, feine interstitielle milch- 


\section{Hier steht eine Anzeige.}

黛 Springer 
glasartige Infiltrate mit einer perihilären Prädominanz gesehen. Die CT zeigt oft diffuse interstitielle und noduläre Parenchymveränderungen [4]. Bei den meisten Patienten ist der LDH-Wert erhöht; dabei sind Werte über 600 oder 700 IE/l mit einer schlechteren Prognose assoziiert [4]. Auskultatorische Befunde sind anfangs minimal [4].

Die Mortalität an PcP bei immunsupprimierten Patienten ohne HIV lag in einer Studie im Zeitraum von 1985-1995 bei $39,4 \%$, bei HIV-Patienten bei 9,4\% [13], eine weitere Publikation aus dem Jahr 2001 bestätigt dieses Verhältnis [20]. Mehrere Erklärungen für diese Differenz wurden diskutiert: Möglicherweise repräsentiere dies Unterschiede zwischen den beiden Patientengruppen in der Interaktion zwischen Wirt und Parasit; möglich sei auch ein Unterschied im Einstrom von Neutrophilen in den alveolären Luftraum. Eventuell sei die Differenz Folge des zugrunde liegenden medizinischen Zustands der HIV-negativen Patienten [13].

\section{Diagnose der PcP}

Während die Untersuchung von Sputa wenig ergiebig ist, können durch die Induktion von Reizsputa mit hypertoner Kochsalzlösung Proben gewonnen werden, die bei 51 HIV-infizierten Patienten eine Sensitivität bezüglich PcP von 94,7\% ergaben [10]. Sputa können mittels Immunfluoreszenz untersucht werden. Kann kein induziertes Sputum gewonnen werden, bzw. persistiert der Verdacht trotz negativer Untersuchung, erbringt eine Bronchoskopie mit Lavage und Untersuchung mit Giemsa-, Toluidinfärbung und Immunfluoreszenz in über $80 \%$ aller Patienten und über 95\% der Patienten mit Aids die Diagnose. Multiple transbronchiale Biopsien erhöhen dabei die diagnostische Ausbeute bei allen Patienten auf über 90\% [3, 4, 17, 23]. Zusätzlich wurde eine PCR-Untersuchung entwickelt [19], die aber nicht routinemäßig eingesetzt wird.

\section{Prophylaxe und Therapie der PcP bei Patienten mit rheumatolo- gischen Erkrankungen}

Singer u. McCune [21] schreiben bezüglich Start einer PcP-Prophylaxe, diese solle sicher bei Initialbehandlung bei WegenerGranulomatose und Vaskulitis eingesetzt werden. In Bezug auf andere rheumatologische Krankheitsbilder sei sie „zu erwägen, wenn potente Immunsuppressiva eingesetzt werden“ [21]. Bei Einsatz von Prednison empfahlen verschiedene Arbeitsgruppen den Einsatz je nach Höhe bzw. Dauer der Behandlung (• Tabelle 1).

Erste Wahl zur PcP-Prophylaxe ist Trimethoprim-Sulfamethoxazol in einer Dosis von 3 Forte-Tabletten (16o/8oo mg)/ Woche. Dabei sollte insbesondere auf das Auftreten einer Leukopenie geachtet werden. Alternativen sind Pentamidin (zur Inhalation mit $300 \mathrm{mg}$ einmal monatlich) und Dapson 2-mal $100 \mathrm{mg} /$ Woche (nach Ausschluss eines G-6-PD-Mangels) [17, 21].

Erste Wahl zur Therapie ist eine Kombination von Dapson $100 \mathrm{mg}$ p.o. einmal täglich mit Trimethoprim 15-20 mg/kg/ Tag über 21 Tage; alternativ Trimethoprim (15-20 mg/kg/Tag) und Sulfamethoxazol (75-100 mg/kg/Tag) p. o. oder i. v. über 21 Tage. Gleich effizient ist die Gabe von Clindamycin/Primaquin. Patienten mit schwerer PcP profitieren von der zusätzlichen Gabe von Prednison in einer Dosis von über $60 \mathrm{mg} / \mathrm{Tag}$ [16].

\section{Synthese anhand des Fallbeispiels}

Der Verlauf bei der vorgestellten Patientin entspricht in mehrfacher Hinsicht dem oben genannten PcP-Verlauf bei rheumatoider Arthritis: Die PcP trat unter wieder gestarteter Methotrexat-Therapie mit zusätzlicher Prednison- und NSAR-Gabe auf. Die am ehesten der PcP zuzurechnende klinische Verschlechterung erfolgte innerhalb weniger Tage, mit fulminantem Verlauf. Ob der Tod direkte Folge der PcP war, ist unklar. Dagegen spräche, dass kurz vor dem Tod keine Pneumozysten mehr in einer Bronchiallavage nachweisbar waren. Welche Rolle der (einmalig nachgewiesene) Aspergillus, die in einer Blutkultur nachgewiesenen Enterokokken und der insgesamt geschwächte Status der Patientin mit vorbestehender Herzinsuffizienz spielten, wurde nicht weiter abgeklärt. Die PcP dürfte aber „Türöffner“ für die zum Tode führenden Komplikationen gewesen sein.

\section{Korrespondierender Autor Prof. Dr. C. Ruef}

Klinik für Infektionskrankheiten und Spitalhygiene, Departement für Innere Medizin, Universitätsspital, Rämistraße 100, 8091Zürich,

E-Mail:christian.ruef@usz.ch

Interessenkonflikt: Es besteht kein Interessenkonflikt. Der korrespondierende Autor versichert, dass keine Verbindungen mit einer Firma, deren Produkt in dem Artikel genannt ist, oder einer Firma, die ein Konkurrenzprodukt vertreibt, bestehen. Die Präsentation des Themas ist unabhängig und die Darstellung der Inhalte produktneutral.

\section{Literatur}

1. Altz-Smith M, Kendall LG Jr, Stamm AM (1987) Cryptococcosis associated with low-dose methotrexate for arthritis. Am J Med 83:179-181

2. Arend SM, Kroon FP, van't Wout JW (1995) Pneumocystis carinii pneumonia in patients without AIDS, 1980 through 1993. An analysis of 78 cases. Arch Intern Med 155:2436-2441

3. Broaddus C, Dake MD, Stulbarg MS et al. (1985) Bronchoalveolar lavage and transbronchial biopsy for the diagnosis of pulmonary infections in the acquired immunodeficiency syndrome. Ann Intern Med 102:747-752

4. Fishman JA (2002) Pneumocystis carinii and parasitic infections in the immunocompromised host. In: Rubin RH, Lowell SY (eds) Clinical approach to infection in the compromised host, 4th edn. Kluwer Academic/Plenum, New York, pp 265-334

5. Godeau B, Coutant-Perronne V, Le Thi Huong D et al. (1994) Pneumocystis carinii pneumonia in the course of connective tissue disease: report of 34 cases. J Rheumatol 21:246-251

6. Kaplan JE, Hanson D, Dworkin MS et al. (2000) Epidemiology of human immunodeficiency virusassociated opportunistic infections in the United States in the era of highly active antiretroviral therapy. Clin Infect Dis 30 Suppl 1:S5-S14

7. Keegan JM, Byrd JW (1988) Nocardiosis associated with low dose methotrexate for rheumatoid arthritis. J Rheumatol 15:1585-1586

8. Kovacs JA, Hiemenz JW, Macher AM et al. (1984) Pneumocystis carinii pneumonia: a comparison between patients with the acquired immunodeficiency syndrome and patients with other immunodeficiencies. Ann Intern Med 100:663-671

9. Krebs S, Gibbons RB (1996) Low-dose methotrexate as a risk factor for Pneumocystis carinii pneumonia. Mil Med 161:58-60

10. Leigh TR, Parsons P, Hume C et al. (1989) Sputum induction for diagnosis of Pneumocystis carinii pneumonia. Lancet 2:205-206

11. LeMense GP, Sahn SA (1994) Opportunistic infection during treatment with low dose methotrexate. Am J Respir Crit Care Med 150:258-260

12. Mansharamani NG, Balachandran D, Vernovsky I et al. (2000) Peripheral blood CD4 + T-lymphocyte counts during Pneumocystis carinii pneumonia in immunocompromised patients without HIV infection. Chest 118:712-720 
13. Mansharamani NG, Garland R, Delaney D, Koziel $H$ (2000) Management and outcome patterns for adult Pneumocystis carinii pneumonia, 1985 to 1995: comparison of HIV-associated cases to other immunocompromised states. Chest 118:704-711

14. Marinosa M, Soler A, Nogues $X$, Pedro-Botet J (2004) Pulmonary coinfection by Pneumocystis carinii and Aspergillus fumigatus in a seronegative arthritis patient treated with low-dose methotrexate. Clin Rheumatol 23:555-556

15. Ognibene FP, Shelhamer JH, Hoffman GS et al. (1995) Pneumocystis carinii pneumonia: a major complication of immunosuppressive therapy in patients with Wegener's granulomatosis. Am J Respir Crit Care Med 51:795-799

16. Pareja JG, Garland R, Koziel H (1998) Use of adjunctive corticosteroids in severe adult non-HIV Pneumocystis carinii pneumonia. Chest 113:1215-1224

17. Rodriguez M, Fishman JA (2004) Prevention of infection due to Pneumocystis spp. in human immunodeficiency virus-negative immunocompromised patients. Clin Microbiol Rev 17:770-782

18. Roux N, Flipo RM, Cortet B et al. (1996) Pneumocystis carinii pneumonia in rheumatoid arthritis patients treated with methotrexate. A report of two cases. Rev Rhum Engl Ed 63:453-456

19. Saito K, Nakayamada S, Nakano K et al. (2004) Detection of Pneumocystis carinii by DNA amplification in patients with connective tissue diseases: re-evaluation of clinical features of $\mathrm{P}$. carinii pneumonia in rheumatic diseases. Rheumatology (Oxford) 43:479-485

20. Sepkowitz KA (2002) Opportunistic infections in patients with and patients without Acquired Immunodeficiency Syndrome. Clin Infect Dis 34:1098-1107

21. Singer NG, McCune WJ (1999) Prevention of infectious complications in rheumatic disease patients: immunization, Pneumocystis carinii prophylaxis, and screening for latent infections. Curr Opin Rheumatol 11:173-178

22. Stenger AA, Houtman PM, Bruyn GA et al. (1994) Pneumocystis carinii pneumonia associated with low dose methotrexate treatment for rheumatoid arthritis. Scand J Rheumatol 23:51-53

23. Stover DE, Zaman MB, Hajdu SI et al. (1984) Bronchoalveolar lavage in the diagnosis of diffuse pulmonary infiltrates in the immunosuppressed host. Ann Intern Med 101:1-7

24. Tai TL, O'Rourke KP, McWeeney M et al. (2002) Pneumocystis carinii pneumonia following a second infusion of infliximab. Rheumatology (Oxford) 41:951-952

25. Ward MM, Donald F (1999) Pneumocystis carinii pneumonia in patients with connective tissue diseases: the role of hospital experience in diagnosis and mortality. Arthritis Rheum 42:780-789

26. Yale SH, Limper AH (1996) Pneumocystis carinii pneumonia in patients without acquired immunodeficiency syndrome: associated illness and prior corticosteroid therapy. Mayo Clin Proc 71:5-13

\section{Wiesemann, N. Billa-Andorno Medizinethik}

Die neue AO

Stuttgart: Thieme 2005, 172 S., (ISBN 3-13-138241-4), 19.00 EUR

Die neue Approbationsordnung für Ärztinnen und Ärzte sieht ab dem Wintersemester 2003/2004 Unterricht im Querschnittsbereich "Geschichte, Theorie, Ethik in der Medizin"

vor.

Die Medizinethik ist damit zu einem prüfungsrelevanten Ausbildungsfach auch im Medizinstudium geworden. Ärztinnen und Ärzte der Zukunft sollen in der Lage sein, kompetent und verantwortungsvoll mit den aus der Medizin resultierenden moralischen Konflikten umzugehen.

Neben der ärztlichen Basis, dem Hippokratischen Eid, sind die rechtlichen Rahmenbedingungen wichtig.

Hier sind das Arzneimittel-Gesetz, das Medizinprodukte-Gesetz, StrahlenschutzVerordnung, die Richtlinien zur guten klinischen Praxis, sowie die Deklaration von Helsinki des Ärztebundes zunennen. Das Menschenrechtsübereinkommen zur Biomedizin des Europarates, der Nürnberger Codex von 1947 müssen ebenfalls bekannt sein.

Das Buch befasst sich darüber hinaus mit Medizinethik und Patienten-ArztBeziehung, Schwangerschaftsabbruch, Reproduktionsmedizin und Embryonenforschung, speziell mit der medizinischen Genetik, der Transplantationsmedizin sowie der Sterbehilfe und Sterbebegleitung.

Ausführungen zur Forschung am Menschen, Tierversuche und der Ausblick auf die öffentliche Gesundheit und Gesundheitsversorgung ergänzen die Broschüre.

Wichtig sind die zahlreichen ergänzenden Hinweise auf weiterführende Literatur, Internetadressen etc..

Lernziele für Medizinethik im Medizinstudium werden im Sinne eines problemorientierten Lernens dargestellt, so dass man über die vielen medizinethisch wichtigen Themen Informationen findet.

Die wichtigsten medizinethischen Fachbegriffe, relevante Richtlinien und
Gesetze sowie Hinweise auf Basisliteratur und websiten werden erfasst, so dass der kleine Band auch für alle Ärzte in der Ausbildung zu empfehlen ist bzw. für Ärzte interessant und wichtig ist, die mit Studien befasst sind.

J. Sökeland (Dortmund) 